\title{
IMPULSE ACTIVITY IN PRESYNAPTIC DENDRITES: ANALYSIS OF MITRAL CELLS IN THE ISOLATED TURTLE OLFACTORY BULB ${ }^{1}$
}

\author{
KENSAKU MORI, ${ }^{2}$ MARTHA C. NOWYCKY, AND GORDON M. SHEPHERD ${ }^{3}$ \\ Sections of Neuroanatomy and Neurosurgery and Department of Physiology, Yale University School of Medicine, \\ New Haven, Connecticut 06510
}

Received August 7, 1981; Revised November 18, 1981; Accepted November 19, 1981

\begin{abstract}
Impulse activity has been reported in neuronal dendrites in several regions of the central nervous system, where it is believed to assist in boosting transmission of signals from remote dendritic sites to the cell body. We have studied this activity in the dendrites of mitral cells in an isolated preparation of the turtle olfactory bulb. Intracellular recordings have been obtained from mitral cells responding to single volleys in the olfactory nerves or lateral olfactory tract. In addition to the large somatic spike, a small fast prepotential (FPP) was present in nearly all cells in response to an orthodromic volley in the olfactory nerves, but it was never seen in antidromic responses from the lateral olfactory tract. Collision tests using antidromic and orthodromic volleys showed that the FPP does not propagate into the axon. Hyperpolarizing current injections caused delay and blocking of the soma spike with little effect on the FPP response. These and other tests provided evidence to localize the FPP in the dendrites and to distinguish it from injury potentials and from spikes in the axon hillock or axonal initial segment.

These results suggest that one function of the impulse in mitral cell dendrites is the classical one of boosting transmission of synaptic responses from the glomerular tuft to the cell body. In addition, it is well established that mitral cell dendrites are presynaptic to the dendrites of interneurons within the bulb and that these connections provide pathways for recurrent inhibition of the mitral cells. It therefore appears that the dendritic impulse in mitral cells acts as a booster for local dendritic synaptic output. These results provide further evidence for the multiple state-dependent inputoutput functions of cells with presynaptic dendrites.
\end{abstract}

Impulse activity has been reported in a number of neuronal dendrites. In most cases, it is believed that this aids in boosting signals from distal dendritic sites to the cell body and axon. A second possible function is to promote local synaptic output in presynaptic dendrites. Although presynaptic dendrites are common in the vertebrate central nervous system (see Pearson, 1976; Schmitt and Worden, 1979; Roberts and Bush, 1981), the only report thus far of spiking activity in these dendrites has been in mitral cells of the rabbit olfactory bulb (Mori and Takagi, 1975).

Mitral cells in the vertebrate olfactory bulb send pri-

\footnotetext{
'This work was supported by Grants NS-07609 and NS-19174 from the National Institute for Neurological and Communicative Disorders and Stroke.

${ }^{2}$ Present address: Department of Physiology, Gunma University School of Medicine, Showa-Machi, Gunma, 371 Japan.

${ }^{3}$ To whom correspondence should be addressed at Section of Neuroanatomy, Yale University School of Medicine, 333 Cedar Street, New Haven, CT 06510.
}

mary dendrites to one or a few glomeruli. In a glomerulus, the primary dendrite arborizes extensively into a tuft of fine branches. A characteristic of olfactory nerve input to mitral cells is that the synapses are localized strictly to the terminal tuft of the primary dendrites, about 300 to $700 \mu \mathrm{m}$ distant from the mitral cell body. In the rabbit, a single volley of impulses in the olfactory nerve elicits in mitral cells an action potential superimposed on an excitatory postsynaptic potential (EPSP) (Yamamoto et al., 1963; Getchell and Shepherd, 1975; Mori and Takagi, 1975). Fast prepotentials have been observed on the rising phase of the action potential, suggesting the presence of active (spiking) membrane in the primary dendrites (Mori and Takagi, 1975).

Recently, we have developed an in vitro preparation of the turtle olfactory bulb (Nowycky et al., 1978; Mori and Shepherd, 1979; Waldow et al., 1981). This isolated preparation permits more stable intracellular recordings from mitral cells, which has provided the opportunity to carry out a more detailed analysis of FPPs. We have reported previously the properties of EPSPs, elicited by 
an olfactory nerve volley, in the mitral cells of the isolated bulb (Mori et al., 1981b). In the present study, we present evidence for the properties of spike potentials generated by these EPSPs at sites in the dendritic tree as well as the cell body, and we discuss the implications of these spikes for dendritic transmission as well as control of local dendritic synaptic output.

\section{Materials and Methods}

The experiments have been carried out on the olfactory bulb of the turtle, Pseudemys scripta. The preparation of the in vitro olfactory bulb and the methods for stimulating and recording have been described fully elsewhere (Mori et al., 1981a). In brief, intracellular recordings reveal stable resting potentials (mean, $-65 \mathrm{mV}$ ) and large antidromic and orthodromic spikes $(60$ to $80 \mathrm{mV}$ ) in mitral cells of the isolated bulb, which can be maintained in good condition for periods of 8 to $12 \mathrm{hr}$. Throughout this period, large amplitude excitatory and inhibitory synaptic potentials can be recorded, including inhibitory potentials lasting for several seconds. In the present study, orthodromic activation was obtained by single electric shocks delivered to the whole olfactory nerve through a suction electrode, while antidromic stimuli were delivered through a bipolar silver wire electrode on the lateral olfactory tract. Intracellular recordings were obtained with $2 \mathrm{M}$ potassium acetate-filled micropipettes, with DC resistances of 40 to 100 megohms. The present report is based on intracellular recordings from 34 cells: 17 antidromically identified mitral cells and 17 presumptive mitral cells (see Mori et al., 1981a, for the method of identification of mitral cells).

\section{Results}

Impulse responses of mitral cells to single olfactory nerve volleys. A single volley in the olfactory nerves (ON) commonly elicited in turtle mitral cells a complex EPSP. When the volley was of adequate strength, the earliest component $\left(\mathbf{E}_{1}\right)$ commonly gave rise to a single impulse (Waldow et al., 1981; Mori et al., 1981b). A typical example is shown in Figure 1A. There was an inflection on the rising phase of this spike (arrow). The effects of progressively increasing hyperpolarizing current injection on the spike response are illustrated in Figure 1, $B$ to $D$. A small amount of current injection (Fig. $1 B$ ) revealed another inflection (open arrow) and caused an enhancement of the first inflection (solid arrow). When more current was injected (Fig. $1 C$ ), the main spike was blocked and a fast prepotential (FPP; arrow) appeared in isolation. The FPP was blocked by a much larger hyperpolarizing current injection as shown in Figure $1 D$.

Another example of FPP generation in mitral cells is shown in Figure 1, $E$ and $F$. A FPP was observed when the main spike was blocked by current injection (lower trace of Fig. $1 E$ ). In Figure $1 F$, the strength of the olfactory nerve volley was adjusted to just threshold for the FPP generation. The superimposed tracings in this figure show that the FPP was generated in an "all-ornothing" manner. This behavior of the FPP contrasts with the graded nature of the underlying synaptic potentials in relation to graded volley strength (Mori et al., 1981b). The above observations indicate that FPPs represent impulse activity rather than synaptic potentials.
FPPs were clearly observed in nearly all (33 out of 34 cells) mitral cells (with or without current injection). A single volley elicited from one to three FPPs with different amplitudes. In about $50 \%$ of the cells, more than one FPP was detected (Figs. $1 B$ and $2 B$ ).

Comparison of orthodromic with antidromic impulses. Figure $2 A$ shows the responses of a mitral cell to lateral olfactory tract (LOT) stimulation. The spike responses had a fixed latency even when progressively increasing amounts of hyperpolarizing current were injected (Fig. 2, $A b$ to $A d$ ), indicating that they were elicited antidromically (see also Mori et al., 1981a). The effect of a hyperpolarizing current injection on this antidromic spike consisted first of an increase in the amplitude of the main spike (Fig. 2, $A b$ and $A c$ ) and then of blocking of the $\mathrm{B}$ spike and isolation of the A spike (Fig. $2 A d)$. In some cells, further increase in the hyperpolarizing current revealed a $M$ spike. The time course and amplitude of the $M$ spike, however, differed from those of the FPP of the orthodromic spike in the same cell. No FPPs could be seen on the rising phase of the antidromic spike.

In Figure 2, $B b$ and $B c$, amounts of hyperpolarizing current similar to those in Figure 2, $A b$ and $A c$, respec-

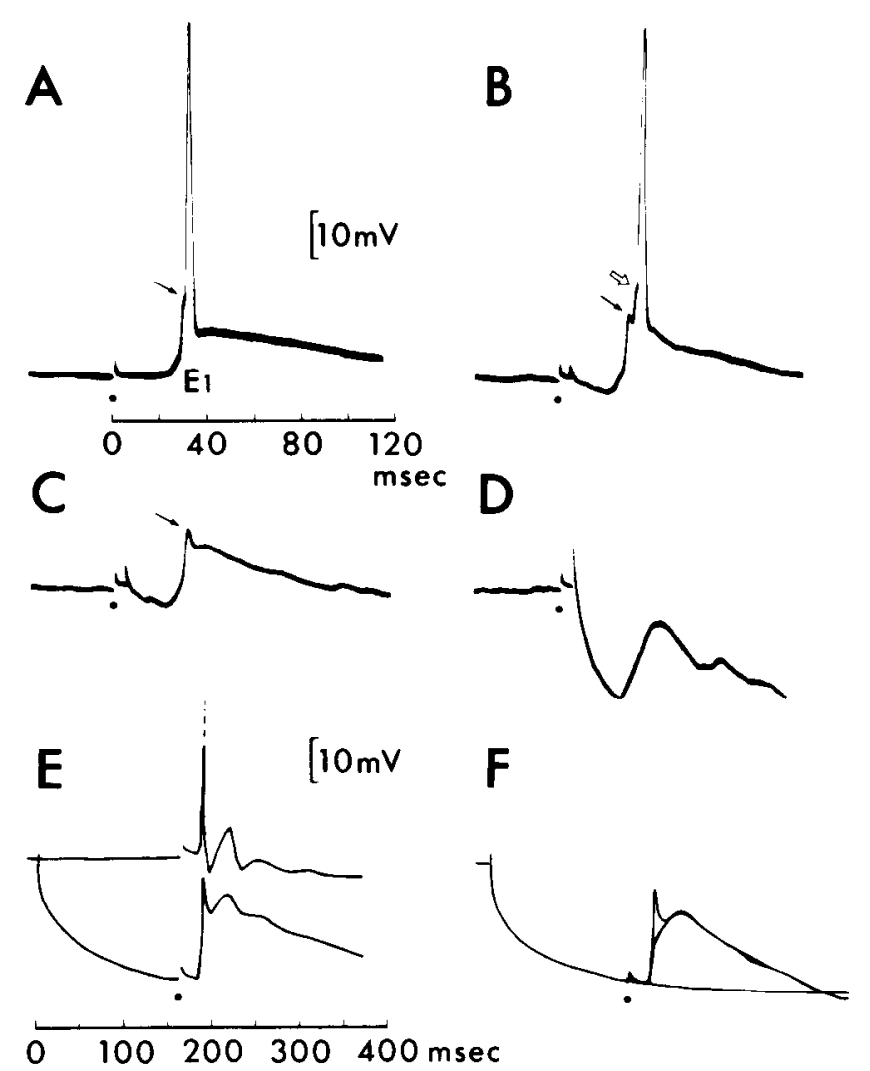

Figure 1. Intracellular responses of mitral cells to olfactory nerve volley. $A$ to $D$, Effect of a hyperpolarizing current injection on the $\mathrm{ON}$-evoked spikes; current intensities: $0.06 \mathrm{nA}$ in $B$, $0.08 \mathrm{nA}$ in $C, 0.50 \mathrm{nA}$ in $D$. , Shock artifact of $\mathrm{ON}$ stimulation; arrows, fast prepotentials; $\mathrm{E}_{1}$, first component of EPSP. $E$ and $F$, Superimposed tracing of the orthodromic responses recorded from another mitral cell. In $F$, the stimulus strength was weakened to just the threshold for the FPP generation. Current intensity: $0.25 \mathrm{nA}$ in $E$ and $F$. 


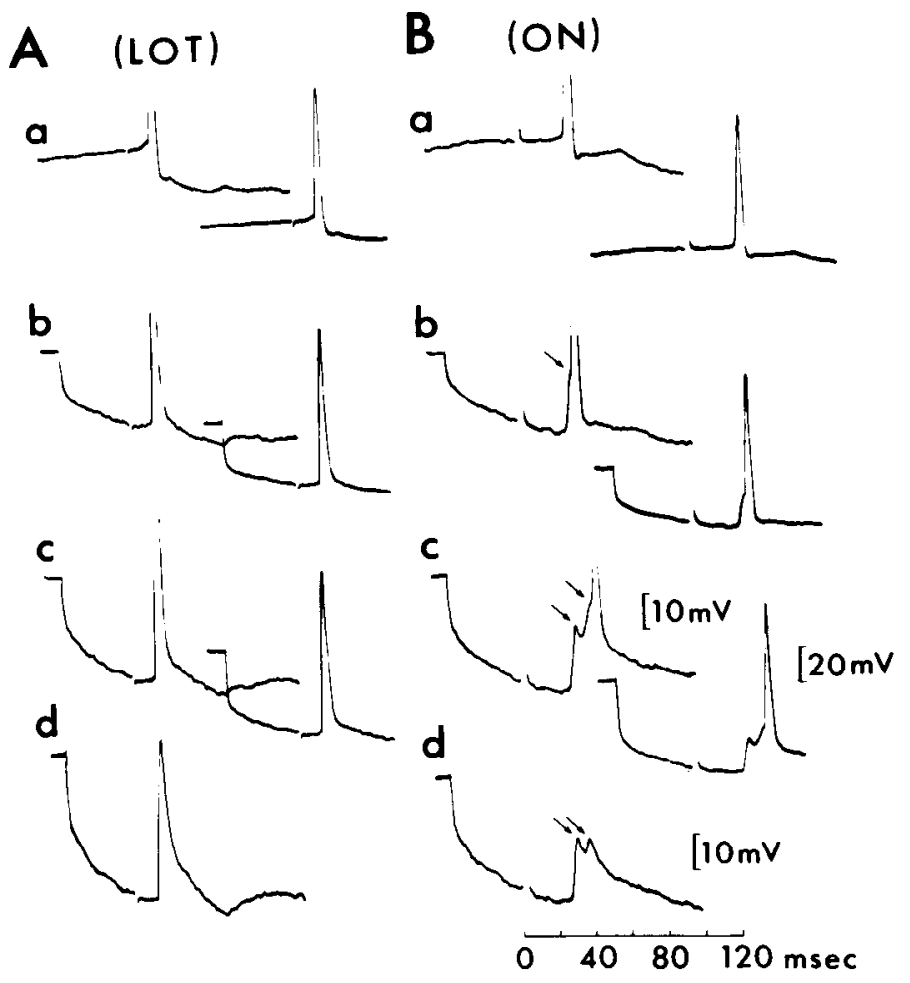

Figure 2. Comparison between antidromic spikes and orthodromic spikes. $A$, Antidromic spikes elicited by LOT stimulation. $B$, Orthodromic spikes evoked by volleys in ON. $A a$ and $B a$ are control responses. $A b$ to $A d$ and $B b$ to $B d$, Effects of hyperpolarizing current $(0.15 \mathrm{nA}$ in $b, 0.20 \mathrm{nA}$ in $c$, and $0.28 \mathrm{nA}$ in $d$ ) on the spike responses. Arrows, fast prepotentials.

tively, were injected. In contrast to the antidromic spike, the same mitral cell showed FPPs on the rising phase of the orthodromic spike. Furthermore, two isolated FPPs were observed when a stronger hyperpolarizing current injection blocked the main spike (Fig. $2 B d$ ). It can be seen that the A spike elicited by LOT stimulation had a larger amplitude and faster rising phase than the FPPs. In 13 cells, the FPPs had amplitudes ranging from 2.5 to $14 \mathrm{mV}$ (mean, $7 \mathrm{mV}$ ), whereas as A spikes varied from 10 to $50 \mathrm{mV}$ (mean, $29 \mathrm{mV}$; see Mori et al., 1981a).

If the FPP were generated in the mitral cell axon, it would travel down the axon to the LOT. Collision tests (Fig. 3) showed that this was not the case. Antidromic and orthodromic spikes are shown in Figure 3, $A$ and $B$, respectively. In Figure $3 C$, this cell was hyperpolarized by a current injection (about $0.2 \mathrm{nA}$ ), and the LOT was stimulated at various latencies following the orthodromic spike. As seen in Figure 3C, antidromic spikes occurred when the interval between the onset of the orthodromic spike and the LOT stimulation was longer than $15 \mathrm{msec}$ (arrows with open circle). This critical interval was very close to the sum of the latency of the antidromic spike $(11 \mathrm{msec})$ and the refractory period of this axon (4.5 msec).

These observations indicate that the main orthodromic spike did, in fact, travel down the axon and collide with an antidromic spike. In Figure $3 D$, the same cell was slightly more hyperpolarized spontaneously and the main orthodromic spike sometimes failed, leaving only a FPP (open arrow). As seen in this figure, the antidromic spike was blocked when the main orthodromic spike was elicited but was unaffected when only the FPP was generated. Similar results were obtained as long as the interval between the onset of the orthodromic spike response and the LOT stimulation was less than $15 \mathrm{msec}$. These results indicate that FPPs do not travel down the mitral cell axon, in contrast to the main orthodromic spike. In most cells, collision testing was obscured by the inhibitory postsynaptic potentials (IPSPs) ( $\mathrm{I}_{1}$ and $\mathrm{I}_{2}$ components) which immediately followed the orthodromic spike. In 3 cells, however, the IPSPs were relatively small, as in Figure 3; results similar to those in Figure 3 were obtained in all of these cells.

Effect of conditioning impulse activity on the FPP. The effects of conditioning spike activity elicited by LOT stimulation or by a depolarizing current injection on the FPP are shown in Figure 4. The mitral cell in Figure $4 A$ responded with a single spike to olfactory nerve stimulation. The inflection between the FPP and the full spike is indicated by an arrow. When an antidromic spike preceded the orthodromic spike, the inflection was accentuated (Fig. $4 B$ ). At a critical interval, the orthodromic full spike was blocked and an isolated FPP was observed (Fig. 4C). The FPP also disappeared with further shortening of the interval between the arrival of the antidromic spike and the orthodromic response (Fig. $4 D$ ). The possibility that this effect was not due to the preceding antidromic spike but caused by inhibitory synaptic potentials elicited by LOT stimulation was checked by the following method. In Figure $4 E$, the orthodromic spike was preceded by an impulse elicited by a brief depolarizing current injection. As shown in Figure 4, $E b$ to $E d$, the preceding spike caused effects similar to those in the case of LOT stimulation in Figure $4, A$ to $D$. They are: (1) accentuation of the inflection (Fig. $4 E b$ ), (2)

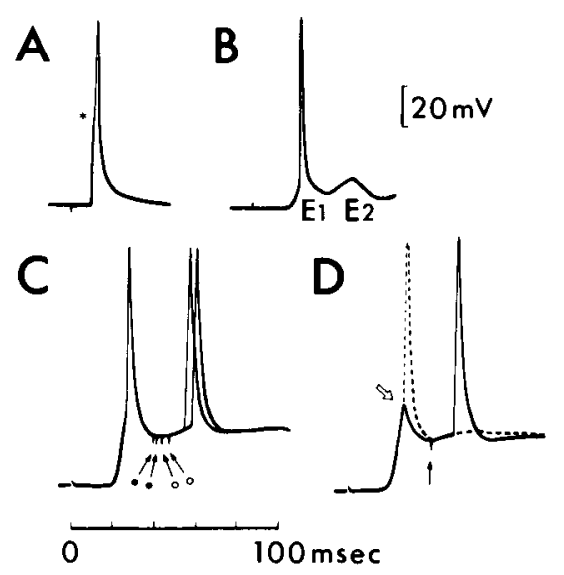

Figure 3. Collision tests between orthodromic spikes and the antidromic spike. $A$, An antidromic spike elicited by LOT stimulation. An A-B inflection was indicated by the asterisk. $B$, Response to $O N$ shock; $E_{1}$ and $E_{2}$ are early and late components, respectively, of EPSP. $C$ and $D$, Response to test LOT stimulation (solid arrows) following conditioning $\mathrm{ON}$ stimulation. Shock artifacts in $C$ are shown at different delays; the later shocks $(O)$ gave rise to the two responses; the early shocks (O) failed to elicit responses. The dotted line in $D$ indicates the responses when ON stimulation elicited a full spike, while the solid line indicates those when $\mathrm{ON}$ stimulation elicited the isolated FPP (open arrow). 

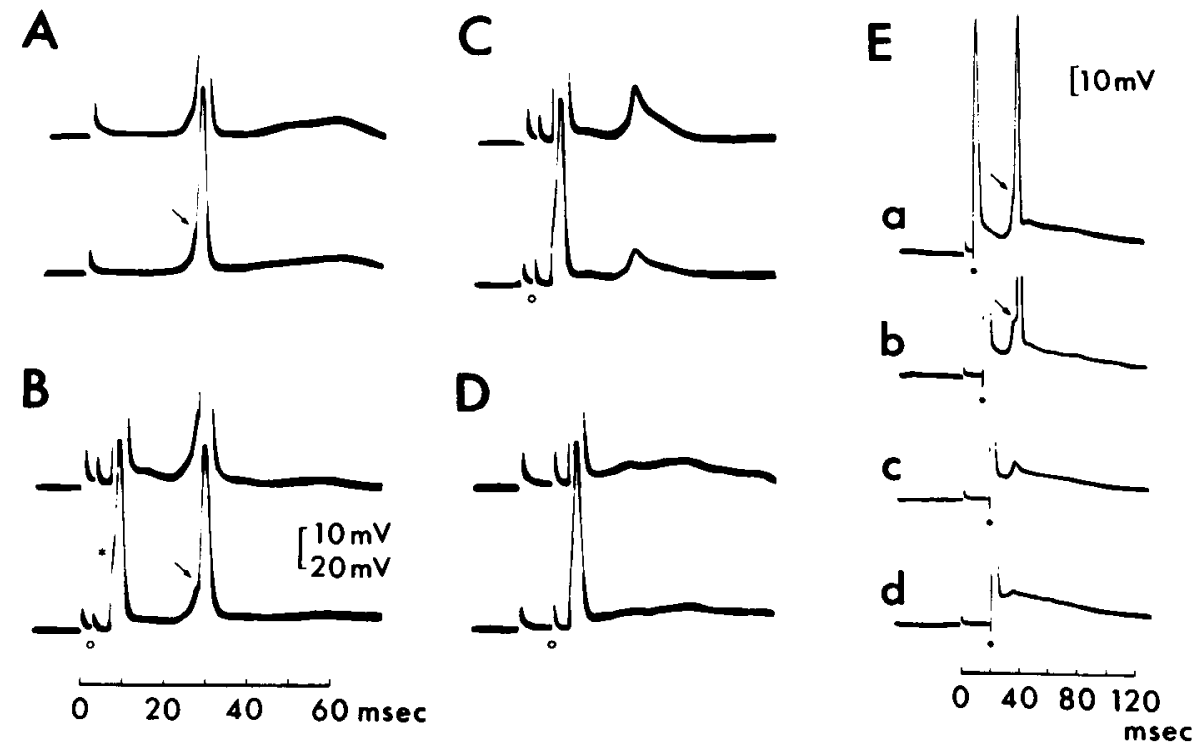

Figure 4. Effects of a conditioning spike on test orthodromic spikes. $A$ to $D$, Conditioning spikes were elicited by a volley in the LO'T. *, A-B inflection; arrow, FPP; $O$, time of LOT stimulation. $E$, Conditioning spikes elicited by depolarizing current injection. Time of onset of the current injection. $E a$ to $E d$ are discussed in the text.

isolation of the FPP (Fig. $4 E c$ ), and finally (3) blocking of the FPP (Fig. $4 E d$ ). In some cells, a preceding spike caused simultaneous blockade of the FPP and the full spike at a critical interval.

Two different modes of spike generation. As reported previously (Mori et al., 1981b), about $15 \%$ of the mitral cells respond consistently with two impulses to a single olfactory nerve volley. An example of this response is shown in Figure 5A. The first and second impulse arose from the $E_{1}$ and $E_{2}$ components, respectively, of the complex EPSP (cf., Mori et al., 1981b). Note that the first spike occurred near the onset of the large positive field potential (upper trace) in agreement with previous studies in the turtle (Mori et al., 1981b) and other species (cf., Shepherd, 1972; Mori and Takagi, 1978). The occurrence of the second spike was correlated with the appearance of a small positive wave on the declining phase of the large field potential, as shown in Figure $5 A$. We shall report elsewhere a detailed analysis of this correlation.

As shown in Figure $5, B$ to $D$, the second spike typically exhibited different behavior from the first spike during the hyperpolarizing current injection. For example, the second spike was easily blocked by a small amount of current injection, whereas the first spike was rather resistant to blockade (Fig. $5 B$ ). In addition, the first spike characteristically arose from an FPP in contrast to the usual lack of an FPP in the second spike (Fig. 5, $B$ to $D$ ). In 7 cells with two impulses, all cells showed FPPs in the rising phase of the first spike when they were hyperpolarized by a current injection, but only 2 cells showed occasional FPPs in the second spike. These results indicate two different sequences of impulse generation in the same mitral cell to olfactory nerve stimulation.

\section{Discussion}

In mitral cells of the isolated turtle olfactory bulb, electrical stimulation of the olfactory nerve elicited the

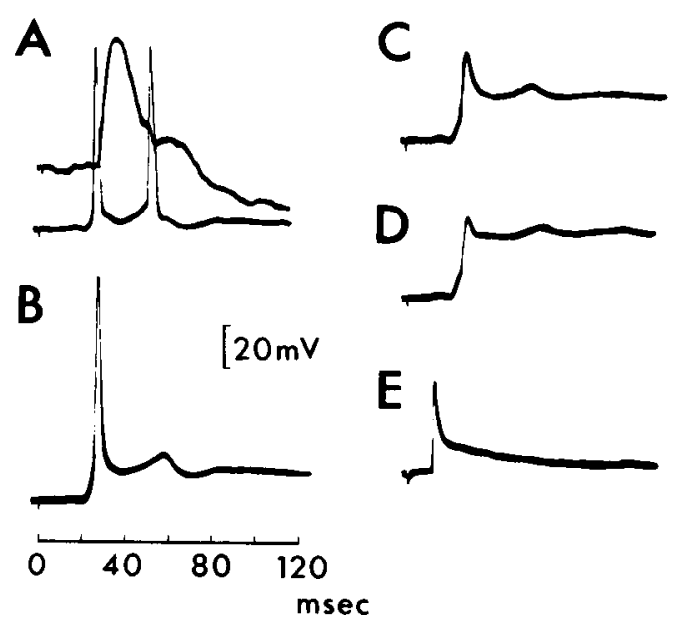

Figure 5. Effects of a hyperpolarizing current injection on action potentials elicited by $\mathrm{ON}$ volley. $A$, Control response. Top trace, field potential recorded in the granule cell layer. Hyperpolarizing currents of $0.13,0.25$, and $0.40 \mathrm{nA}$ were injected in $B, C$, and $D$, respectively. $E$, An antidromically elicited $A$ spike following LOT stimulation when a hyperpolarizing current of $0.40 \mathrm{nA}$ was injected.

full somatic spike in two different ways (cf., Fig. 5). One was a sequence of EPSP ( $E_{1}$ component)-FPPs-full spike. The other was EPSP ( $\mathrm{E}_{2}$ component)-full spike without accompanying FPPs. In this study, we have mainly analyzed the former mode of orthodromic activation of mitral cells. The $\mathrm{E}_{2}$ components (also termed long lasting EPSPs) are discussed elsewhere (Nowycky et al., 1981a, b).

The analysis has shown that FPPs in turtle mitral cells are action potentials which are not generated in the initial or myelinated segment of the axon. Collision testing with antidromic spikes indicated that, when FPPs 
were elicited without an accompanying full spike, they did not lead to a conducted action potential in the mitral cell axon. These results and the small amplitude of the FPPs suggest that they are active spikes generated in the dendritic membrane.

It also seemed possible, however, that FPPs represent local responses generated at patches in the somatic membrane. In fact, we sometimes observed small abortive spikes superimposed on the top of antidromic A spikes during blockade of the $\mathrm{B}$ spike by a hyperpolarizing current injection. Partial spikes resembling the FPPs also were observed in presumably damaged mitral cells (judged by their low resting membrane potential). Partial spikes occurred spontaneously or were elicited by a depolarizing current injection. These small abortive spikes and partial spikes differed from FPPs in being easily blocked by a hyperpolarizing current injection; they may have been generated in small parts of the active membrane of the soma or proximal dendrites which were damaged by the micropipette insertion.

Several further properties of the FPPs differed from those of the small abortive or partial spikes. (1) FPPs elicited by $\mathrm{ON}$ stimulation were quite resistant to hyperpolarizing current injection. (2) FPPs were elicited only by ON stimulation. No FPP was observed following LOT stimulation or depolarizing current injection. (3) FPPs were observed in virtually all of the mitral cells with high resting membrane potential. (4) The amplitude and time course of FPPs were relatively constant throughout the recording, while those of the abortive spikes or partial spikes sometimes changed with changes in the resting membrane potential. These properties of FPPs indicate that they are not local responses in the soma or proximal dendrites.

Since FPPs are always observed on the EPSP $\left(\mathrm{E}_{1}\right.$ component) elicited by $\mathrm{ON}$ stimulation, the primary dendrites seem to be responsible for the generation of the FPPs. The small amplitude and relatively slow rising phase of the FPP, together with the relatively effective passive spread of electrotonic potentials in mitral cell dendrites (Mori et al., 1981a), suggest that the FPP generation sites may be located in rather distal portions of the primary dendrites. Further experiments (especially using recordings from dendrites) are necessary to clarify this point.

Fast prepotentials or small active spikes which were interpreted as dendritic spikes have been reported in a number of neurons in the central nervous system (e.g., hippocampal pyramidal cells (Spencer and Kandel, 1961; Schwartzkroin, 1977), immature cortical cells (Purpura et al., 1965), and chromatolytic motoneurons (Eccles et al., 1958; Kuno and Llinas, 1971)). Spikes were recorded directly from the dendrites of Purkinje cells by Llinas and Nicholson (1971) and Llinas and Sugimori (1980) and hippocampal pyramidal cells by Wong et al. (1979). In both of these regions, recordings showed multiple spike-generating sites in the dendrites.

In the turtle olfactory bulb, we frequently have observed more than one FPP in a single mitral cell. This indicates that turtle mitral cells also have multiple sites of FPP generation. It is interesting to note that turtle mitral cells have more than one primary dendrite (usually two), each of which projects to different glomeruli (Or- rego, 1961; Mori et al., 1981a), in contrast to a single primary dendrite connecting to a single glomerulus in rabbit mitral cells. This might be related to the higher incidence of multiple FPPs in turtle mitral cells compared with the rabbit mitral cells.

Functional significance. We conclude that turtle mitral cells, like rabbit mitral cells and a variety of other types of neuron, have the ability to generate action potentials at sites different from that responsible for generation of the spike at the cell body-axon hillock region. The present results do not indicate the site or sites at which this activity may occur. If we can rule out the axon (as could be done for at least some cases), we are left with several possible sites in the dendritic tree. The most likely sites are the junction of the primary and secondary dendrites, the distal segment of the primary dendrite where it gives rise to the terminal tuft, and within the glomerular tuft itself. We are presently developing computer simulations of the turtle mitral cell to test these possibilities.

Dendritic spikes may be significant in relation to several functions in mitral cells. One function is to serve as a spike booster so that remote EPSPs in the glomerular tuft can activate full spikes in the cell body or initial segment. This is the classical function ascribed to fast prepotentials in hippocampal pyramidal cells by Spencer and Kandel (1961). A second and related function is to serve as a local site of synaptic integration. Mitral cells receive many different inputs in the glomerular tuft and primary dendrites. In addition to excitatory inputs from olfactory nerves, there are inhibitory inputs from dendritic and axon terminals of a type of periglomerular cell (Getchell and Shepherd, 1975; Ribak et al., 1977). There are also inputs from centrifugal fibers and long lasting excitatory synaptic inputs of unknown origin (Nowycky et al., 1981b). Dendritic spikes in the distal portion of the mitral cell primary dendrite thus represent the integrated outcome of complex synaptic interactions which may occur, to a large extent, independently of the functional state of the soma or proximal dendrites.

Another important function of FPPs in mitral cells is in relation to dendritic synaptic output. The mitral cell is presynaptic as well as postsynaptic throughout most of its dendritic branching structure (see Rall et al., 1966; Price and Powell, 1970; Reese and Shepherd, 1971; Pinching and Powell, 1971). These output synapses may be activated or inhibited by the variety of synaptic inputs described above. In addition, any spike activity in the dendrites is in a position to activate directly this local dendritic output. Thus, dendritic spikes in presynaptic dendrites may serve as boosters for local synaptic output from the dendrites as well as for distant impulse output through the axon. We have reported previously evidence for calcium spikes in the soma of the mitral cell and have discussed their importance for the control of synaptic output from the soma and dendritic trunks; calcium movements might have similar roles in association with dendritic spiking activity (Mori et al., 1981a).

Under conditions of weak afferent stimulation combined with inhibition of the soma, the dendritic active site or sites can operate independently of the generation of full spikes, thus giving rise to complex integration and local synaptic output independent of axonal output. With 
stronger afferent input, the dendritic booster spike is linked more closely to full spike production. This is clearly seen in our present results. Dendritic spike activity thus provides further evidence for multiple statedependent input-output functions of cells with presynaptic dendrites (Shepherd, 1979, 1981). The local inputoutput functions will depend on the specific types of interneurons with which the mitral cell interacts at different loci on its dendritic tree. Further studies are needed to discriminate between these actions of different interneurons in the olfactory bulb and in other regions of the nervous system in which presynaptic dendrites and dendritic spikes are found.

\section{References}

Eccles, J. C., B. Libet, and R. R. Young (1958) The behavior of chromatolysed motoneurons studied by intracellular recordings. J. Physiol. (Lond.) 143: 11-40.

Getchell, T. V., and G. M. Shepherd (1975) Synaptic actions in mitral and tufted cells elicited by olfactory nerve volleys in the rabbit. J. Physiol. (Lond.) 251: 497-522.

Kuno, M., and R. Llinas (1971) Enhancement of synaptic transmission by dendritic potentials in chromatolyzed motoneurons of the cat. J. Physiol. (Lond.) 210: 807-821.

Llinas, R., and C. Nicholson (1971) Electrophysiological properties of dendrites and somata in alligator Purkinje cells. J. Neurophysiol. 34: 532-551.

Llinas, R., and M. Sugimori (1980) Electrophysiological properties of in vitro Purkinje cell dendrites in mammalian cerebellar slices. J. Physiol. (Lond.) 30.5: 197-213.

Mori, K., and G. M. Shepherd (1979) Synaptic excitation and long-lasting inhibition of mitral cells in the in vitro turtle olfactory bulb. Brain Res. 172: 155-159.

Mori, K., and S. F. Takagi (1975) Spike generation in the mitral cell dendrite of the rabbit olfactory bulb. Brain Res. 100: $685-689$.

Mori, K., and S. F. Takagi (1978) An intracellular study of dendrodendritic inhibitory synapses on mitral cells in the rabbit olfactory bulb. J. Physiol. (Lond.) 279: 569-588.

Mori, K., M. C. Nowycky, and G. M. Shepherd (1981a) Electrophysiological analysis of mitral cells in isolated turtle olfactory bulb. J. Physiol. (Lond.) 314: 281-294.

Mori, K., M. C. Nowycky, and G. M. Shepherd (1981b) Analysis of synaptic potentials in mitral cells of the isolated turtle olfactory bulb. J. Physiol. (Lond.) 314: 295-309.

Nowycky, M. C., U. Waldow, and G. M. Shepherd (1978) Electrophysiological studies in isolated turtle brain. Soc. Neurosci. Abstr. 4: 583.

Nowycky, M. C., K. Mori, and G. M. Shepherd (1981a) GABAergic mechanisms of dendrodendritic synapses in isolated turtle olfactory bulb. J. Neurophysiol. 46: 639-648.
Nowycky, M. C., K. Mori, and G. M. Shepherd (1981b) Blockade of synaptic inhibition reveals long-lasting synaptic excitation in isolated turtle olfactory bulb. J. Neurophysiol. 46: 649-658.

Orrego, F. (1961) The reptilian forebrain. I. The olfactory pathways and cortical area in the turtle. Arch. Ital. Biol. 99: 425-445.

Pearson, K. (1976) Nerve cells without action potentials. In Simpler Networks and Behavior, J. C. Fentress, ed., pp. 99110, Sinauer Associates, Sunderland, MA.

Pinching, A. J., and T. P. S. Powell (1971) The neuropil of the glomeruli of the olfactory bulb. J. Cell Sci. 9: 347-377.

Price, J. L., and T. P. S. Powell (1970) The synaptology of the granule cells of the olfactory bulb. J. Cell Sci. 7: 125-155.

Purpura, D. P., R. J. Shofer, and T. Scarff (1965) Properties of synaptic activities and spike potentials of neurons in immature neocortex. J. Neurophysiol. 28: 925-942.

Rall, W., G. M. Shepherd, T. S. Reese, and M. W. Brightman (1966) Dendrodendritic synaptic pathway for inhibition in the olfactory bulb. Exp. Neurol. 14: 44-56.

Reese, T. S., and G. M. Shepherd (1971) Dendro-dendritic synapses in the central nervous system. In Structure and Function of Synapses, G. D. Pappas and D. P. Purpura, eds., pp. 121-136, Raven Press, New York.

Ribak, C. E., J. E. Vaughn, K. Saito, R. Barber, and E. Roberts (1977) Glutamate decarboxylase localization in neurons of the olfactory bulb. Brain Res. 126: 1-18.

Roberts, A., and B. M. H. Bush, eds. (1981) Neurons Without Impulses. Society for Experimental Biology Seminar Series, Vol. 6, Cambridge University Press, Cambridge, England.

Schmitt, F. O., and F. G. Worden (1979) The Neurosciences: Fourth Study Program, MIT Press, Cambridge, MA.

Schwartzkroin, P. A. (1977) Further characteristics of hippocampal CA cells in vitro. Brain Res. 128: 53-68.

Shepherd, G. M. (1972) Synaptic organization of the mammalian olfactory bulb. Physiol. Rev. 52: 864-917.

Shepherd, G. M. (1979) The Synaptic Organization of the Brain, Ed. 2, Oxford University Press, New York.

Shepherd, G. M. (1981) The nerve impulse and the nature of nervous function. Soc. Exp. Biol. Semin. Ser. 6: 1-27.

Spencer, W. A., and E. R. Kandel (1961) Electrophysiology of hippocampal neurons. IV. Fast prepotentials. J. Neurophysiol. 24: 272-285.

Waldow, U., M. C. Nowycky, and G. M. Shepherd (1981) Evoked potential and single unit response to olfactory nerve volleys in the isolated turtle olfactory bulb. Brain Res. 211: 267-283.

Wong, R. K. S., D. A. Prince, and A. I. Basbaum (1979) Intradendritic recordings from hippocampal neurons. Proc. Natl. Acad. Sci. U. S. A. 76: 986-990.

Yamamoto, C., T. Yamamoto, and K. Iwama (1963) The inhibitory systems in the olfactory bulb studied by intracellular recordings. J. Neurophysiol. 26: 403-415. 